\title{
Zum Gedenken an Professor Gerhard Furrer, 26. Februar 1926-10. September 2013
}

\section{H. Elsasser}

\section{Gedenkfeier vom 25. September 2013}

Am 30. September 1989 hielt Gerhard Furrer anlässlich der Feier zum Jubiläum 100 Jahre GeographischEthnographischen Gesellschaft Zürich einen Vortrag über die Entwicklung der Zürcher Geographie. Darin schilderte er sehr lebendig, wie er zur Geographie kam: Bei einem Sonntagsspaziergang im Zürcher Unterland erklärte ihm sein Vater, gestützt auf Untersuchungen von Professor Albert Heim, dass die Hügel der Periglaziallandschaft zwischen Stadel und Glattfelden ein Gletscher aufgestossen habe. Gerhard wagte gegen diese Autoritäten keinen Widerspruch, keine Zweifel anzumelden - denn er sah und fand die Gletscher nicht.

1941 kam Gerhard nach Schiers. Die Evangelische Lehranstalt wurde für fünf Jahre seine Heimat. „Dort im Prättigau reifte mein Entschluss zum Geographiestudium. Einerseits wollte ich erfahren, ob Vater und Professor Heim mit ihren Gletschern recht behielten ... Anderseits begann mich die alpine Kulturlandschaft zu faszinieren, das Resultat des Zusammenspiels von Mensch und Natur, das ich während meines obligatorischen Landdienstes bei einem Prättigauer Bergbauern zu ahnen begann."

Nach dem Studium der Geographie an der Universität Zürich, mit einer Diplomarbeit über das Furttal und Promotion über Solifluktionsformen im Schweizerischen Nationalpark, folgte - immer wieder unterbrochen durch Militärdienst die Ausbildung zum Gymnasiallehrer. 1957 wurde Geri Furrer zum Hauptamtlichen Geographielehrer am Realgymnasium Zürich gewählt. Ich hatte das Glück zu seinen ersten Schülern am Realgymnasium zu zählen. Sein Unterricht war engagiert, spannend; er war ein glänzender Geschichtenerzähler. Und Geri Furrer war, was uns Schüler sehr erfreute, für die damalige Zeit teilweise recht unkonventionell. Wir lernten in seinem Unterricht selbständig zu denken und $\mathrm{Zu}$ sammenhänge zu erkennen; Auswendiglernen war bei ihm weniger wichtig. Er konnte uns für die Geographie oder zumindest für einzelne Teilaspekte des Fachs begeistern. Neben schulischen Fragen interessierte sich Geri Furrer immer auch für die einzelnen Schüler mit ihren persönlichen Problemen.
Der Schüler stand bei ihm im Zentrum des Unterrichts. Auch als Hochschullehrer hatte er immer ein offenes Ohr für Probleme der Studierenden und fand in vielen Fällen gute Lösungen.

Verschiedene seiner Gymnasiasten traf er später wieder als Geographiestudenten an der Universität. Die Beziehungen zwischen Mittelschule und Universität waren ihm immer sehr wichtig. Der Kontakt zu seinen Geographielehrerkollegen brach nie ab, und er verfolgte über seine Emeritierung hinaus die Entwicklungen im Schulfach Geographie mit regem Interesse. Er erkannte aber auch früh, dass Berufsfelder ausserhalb der Schule für das Fach Geographie immer wichtiger wurden, und dass sich die Ausbildung der Geographiestudierenden entsprechend ausrichten musste. Für ihn, den ehemaligen Geographielehrer, waren Schulgeographie und Angewandte Geographie keine Gegensätze.

Parallel zu seiner Tätigkeit als Gymnasiallehrer verfasste Geri Furrer seine Habilitationsschrift und wurde 1965 Privatdozent an der damaligen Philosophischen Fakultät II der Universität Zürich. Die Feldarbeiten für seine Habilitationsschrift führten ihn nach Ostspitzbergen sowie ins Braldo- und Biafotal im Karakorum. Dort untersuchte er die subnivalen Bodenformen, speziell ihre Höhenlage, und zog Vergleiche zu den Verhältnissen in den Bündner und Walliser Alpen. Geri Furrer liebte die Feldarbeit, er besuchte gerne seine Diplomandinnen und Diplomanden, seine Doktorandinnen und Doktoranden im Felde um mit ihnen vor Ort wissenschaftlich zu diskutieren. Im Zusammenhang mit der Feldarbeit müssen auch seine zahlreichen Exkursionen sowie seine legendäre Spitzbergenexpedition und die Expedition auf den Kilimanjaro genannt werden. Neben der Feldarbeit förderte er aber auch die Arbeit im Labor. Er führte zusammen mit seinen Mitarbeitern beispielsweise die Radiocarbondatierung sowie die Pollen- und Schwermetallanalyse am Geographischen Institut ein.

1968 wurde er zum Extraordinarius ernannt und fünf Jahre später zum Ordinarius befördert. Geri Furrer vertrat am Geographischen Institut die Physische Geographie. In der Forschung rückte ab den siebziger Jahren die Paläogeographie, 
die Gletschergeschichte und damit verbunden die Klimageschichte vermehrt in den Vordergrund. Zusammen mit seinem Team leistete er wichtige wissenschaftliche Beiträge zur aktuellen Klimadiskussion. Dabei pflegte er einen engen Kontakt zu anderen Geographischen Instituten in der Schweiz und im benachbarten Ausland, insbesondere in Deutschland und in Österreich.

Neben der Physischen Geographie interessierte ihn aber auch die Anthropogeographie. So veröffentlichte er beispielsweise bereits in den sechziger Jahren in der bekannten Zeitschrift für Agrargeschichte und Agrarsoziologie eine siedlungs- und agrargeographische Untersuchung über das schon genannte Braldotal. Unter seiner Leitung entstanden Dissertationen und Diplomarbeiten zur Siedlungs- und Wirtschaftsgeographie von Walsersiedlungen, über die Bewässerung im Münstertal oder zu Heiratskreisen.

Das Hauptforschungsgebiet von Geri Furrer war - räumlich gesehen - der Kanton Graubünden. Schon als Mittelschullehrer vermochte er eine grosse Zahl von Gymnasiasten für die Schönheiten dieses Alpenkantons zu begeistern, aber auch für dessen natur- und kulturräumliche Probleme zu sensibilisieren. Eine Vielzahl von Geographinnen und Geographen arbeitete unter seiner Leitung und Betreuung in Graubünden. Dank seiner Forschungsarbeiten und -impulse hat sich das Geographische Institut an der Universität Zürich zu einer eigentlichen Geographischen Forschungsstelle für den Kanton Graubünden entwickelt. Er versuchte aber nie die geographischen Forschungen in Graubünden zu monopolisieren. Eine grosse Freude für Geri Furrer war stets, wenn auch an anderen Hochschulinstituten im In- und Ausland über Graubünden gearbeitet wurde und wenn er dank seiner reichen Erfahrungen und seiner Beziehungsnetze diese Forschungen mit Rat und Tat unterstützen konnte. Die Festschrift zu seinem 65. Geburtstag 1991 trägt deshalb auch den Titel „Beiträge zur Geographie Graubündens“.

Für seine Forschungsaktivitäten sowie auch für seinen Einsatz für die Geographie erhielt Geri Furrer zahlreiche Ehrungen: Mitglied der Akademie für Wissenschaft und Literatur Mainz, korrespondierendes Mitglied der Frankfurter Geographischen Gesellschaft, Ehrenmitglied der Deutschen Quartär Vereinigung, der Schweizerischen Geomorphologischen Gesellschaft sowie der GeographischEthnographischen Gesellschaft Zürich. Die grösste Freude bereitete ihm aber eine andere Ehrung, die Ernennung zum Ehrenpräsidenten der Stiftung Fledermausschutz Schweiz.
Nach dem unerwarteten Tod von Professor Hans Boesch 1978 wurde die Leitung des Geographischen Instituts Geri Furrer übertragen. In seine Zeit als Institutsdirektor fiel 1983 der Wechsel vom Standort Blümlisalpstrasse zum Standort Irchel. Geri Furrer war für diesen Standortwechsel, der mit einem bedeutenden Ausbau des Instituts verbunden war, der richtige Mann zur richtigen Zeit am richtigen Ort. Er hat mit seiner Idee eines hinsichtlich Forschung und Lehre breit angelegten Instituts sowie seinem Durchsetzungsvermögen ganz wesentlich zur Stärkung der Geographie beigetragen. Er hat nie den Gesamtblick für die Geographie verloren und hat sich als Physischer Geograph mit Erfolg stets gegen eine Aufspaltung und eine Aufteilung der Geographie auf verschiedene Fakultäten gewandt.

Auf die Zeit als Institutsdirektor folgte die Zeit als Dekan der Philosophischen Fakultät II (1985-1987). Institutsdirektion und Dekanat waren für Geri Furrer nicht Pflichtaufgaben, die es zu erfüllen gab, sondern es war seine Kür, die er mit Freude und Spass absolvierte. Vielen bleibt sein Fakultätsausflug mit Nachtessen und Sondervorstellung im Zirkus Monti in Erinnerung. In seiner Arbeit als Institutsdirektor und Dekan war er stets sehr gut organisiert. Eine seiner Stärken war ferner, dass er über sich selber lachen konnte.

Der bereits genannte Vortrag von Gerhard Furrer schliesst sinngemäss mit folgenden Aussagen: Der Mensch ist nicht Beherrscher der Natur, sondern Teil der Natur. Seine Aufgabe besteht darin, zu bewahren und zu pflegen, statt zu herrschen. Die Richtlinien für das konkrete praktische Handeln des Menschen heissen: Ehrfurcht, Bescheidenheit und Rücksicht. Für uns Geographen ergibt sich eindeutig die Verantwortung, die wir als Forscher und als Menschen erfüllen müssen im Lebensgefüge von Landschaft und Mensch.

Gerhard Furrer war sich seiner Verantwortung bewusst und hat als Gymnasiallehrer, Hochschullehrer und Forscher entsprechend nachhaltig gehandelt. 